Décadrages Décadrages

cınéma, à travers champs Cinéma, à travers champs

$15 \mid 2009$

Raoul Ruiz

\title{
De Proust à Ruiz, reconnaissance dans Le Temps retrouvé
}

\section{Alain Freudiger}

\section{(2) OpenEdition}

1 Journals

Édition électronique

URL : https://journals.openedition.org/decadrages/117

DOI : $10.4000 /$ decadrages. 117

ISSN : 2297-5977

Éditeur

Association Décadrages

Édition imprimée

Date de publication : 1 octobre 2009

Pagination : 69-77

ISBN : 978-2-9700668-0-4

ISSN : 2235-7823

\section{Référence électronique}

Alain Freudiger, "De Proust à Ruiz, reconnaissance dans Le Temps retrouvé », Décadrages [En ligne], 15 | 2009, mis en ligne le 26 novembre 2012, consulté le 03 avril 2022. URL : http://

journals.openedition.org/decadrages/117 ; DOI : https://doi.org/10.4000/decadrages.117 


\section{De Proust à Ruiz, reconnaissance dans}

\section{Le Temps retrouvé}

\section{par Alain Freudiger}

1 Marcel Proust, Le Temps retrouvé, Gallimard (Folio), 1972 [1927], p. 310.

Au tout début du Temps retrouvé de Raoul Ruiz (France/Portugal/Italie, 1998), une séquence nous montre Marcel, dans son lit, regardant tour à tour une série de photographies représentant différents personnages. Il les nomme, il les reconnait: Gilberte, Maman, Papa, Cottard... Cette séquence a plusieurs fonctions, dont bien évidemment celle de "présentation» des protagonistes du récit; mais cette fonction-là, la plus évidente, n'est pourtant pas la principale. Pour preuve, plusieurs des personnages, notamment Cottard et Papa, n'apparaitront quasiment pas au cours du film. A l'inverse, d'autres personnages très présents, comme Morel, ou Charlus, ne figurent pas sur les photographies. C’est que cette séquence a surtout pour fonction de présenter Marcel lui-même, à la fois comme le narrateur et le remémorant; les photos, elles, ne servent que de support : c'est le souvenir qui est en jeu; et, aussi bien, la reconnaissance.

Cet article se propose non pas d'analyser le film, ni de traiter directement de l'adaptation de Proust par Ruiz, mais plus modestement d'étudier cette question à la fois centrale et d'arrière-plan, celle de la reconnaissance.

\section{La question de la reconnaissance chez Proust}

A de multiples reprises, dans l'ensemble d'A la recherche du Temps perdu, et particulièrement dans $A$ l'ombre des jeunes filles en fleur et Le Temps 
retrouvé, Proust met en jeu la question de la reconnaissance, reconnaissance non pas au niveau social - bien que cela entre aussi en jeu - mais, plus fondamentalement, reconnaissance d'une personne au niveau de sa physionomie, ou de son identité. Or, qu'est-ce que "reconnaître» quelqu'un? C'est relier des traits, singulièrement ceux du visage - on sait l'importance du visage dans l'identité d'une personne et dans sa reconnaissance par une autre : le visage est un signifiant maître, lieu dans la prime enfance de la reconnaissance la plus primordiale, comme l'a montré la psychanalyse -, à une identité, fixée pour le "reconnaissant» dans un autre temps et éventuellement un autre lieu. Cette identité peut d'ailleurs être fixée par des moyens techniques: l'essor du portrait photographique, à cette époque, est également celui du portrait exécuté (photo d'identité, anthropométrie) - tous deux étant liés à la mort, que ce soit pour transmettre une identité au-delà, ou pour dresser un portrait définitif. Reconnaître se joue ainsi sur plusieurs axes, et d'abord sur celui, strictement physionomique, de la reconnaissance des traits: il s'agit alors de détailler des traits isolés tout en essayant de les constituer en un ensemble, de les séparer pour les unir en une perception globale qui serait plus précise ou irait plus loin que la perception primaire. Cet exercice est en soi déjà difficile, comme Proust l'évoque à de multiples reprises, du fait du caractère non figé d'un visage, de sa motilité (moues, plasticité), de son évanescence - ou même d'une individuation encore non effectuée :

«Elles n'étaient plus loin de moi. Quoique chacune fût d'un type absolument différent des autres, elles avaient toutes de la beauté; mais, à vrai dire, je les voyais depuis si peu d'instants et sans oser les regarder fixement que je n'avais encore individualisé aucune d'elles. Sauf une, que son nez droit, sa peau brune mettaient en contraste au milieu des autres comme, dans quelque tableau de la Renaissance, un roi Mage de type arabe, elles ne m'étaient connues, l'une que par une paire d'yeux durs, butés et rieurs; une autre que par des joues où le rose avait cette teinte cuivrée qui évoque l'idée de géranium; et même ces traits, je n'avais encore indissolublement attaché aucun d'entre eux à l'une des jeunes filles plutôt qu'à l'autre; et quand (selon l'ordre dans lequel se déroulait cet ensemble, merveilleux parce qu'y voisinaient les aspects les plus différents, que toutes les gammes de couleurs y étaient rapprochées, mais qui était confus comme une musique où je n'aurais pas su isoler et reconnaître au moment de leur passage les phrases, distinguées mais oubliées aussitôt après) je voyais émerger un ovale blanc, des yeux noirs, des yeux verts, je ne savais pas si c'était les mêmes qui m’avaient déjà apporté 
du charme tout à l'heure, je ne pouvais pas les rapporter à telle jeune fille que j'eusse séparée des autres et reconnue. Et cette absence, dans ma vision, des démarcations que j'établirais bientôt entre elles, propageait à travers leur groupe un flottement harmonieux, la translation continue d'une beauté fluide, collective et mobile.» $\mathbf{2}$

A ce premier axe s'ajoute un autre: il faut relier cette perception à une perception antérieure, une image, un souvenir - celui-ci peut être très ancien ou tout récent, mais cette autre image, avant de se constituer, de finir par se fixer, se figer, a souffert des mêmes difficultés que la première (perception globale et motilité) - d'où un décalage forcé avec la perception présente où va se jouer la "reconnaissance", puisque sans même parler du temps écoulé (changements liés au vieillissement) ou des conditions du souvenir (focalisation sur un détail, négligence d'un aspect, artifice - maquillage, déguisement), une image fixée ne peut être que "décevante» (trompeuse) dans sa confrontation au vivant d'un visage. L'union se fait alors par le recours à une identité, qui est d'un autre registre, peut-être plus abstrait, subsumée par un nom propre, et qui est toujours aussi un processus d'identification qui implique le regardant, et sa place par rapport à l'autre. Cela est d'ailleurs frappant à la fois chez Proust, où le titre de «duchesse de Guermantes» recouvre la personne physique d'Oriane - et le narrateur évoque à diverses reprises sa difficulté à faire coïncider les deux - et chez Ruiz, où les rôles sont des stéréotypes vides dans lesquels, par bribes, des personnages mouvants viennent ou non s'incarner - nous verrons plus loin l'importance du spectateur (et donc d'un autre regardant) dans ce processus. Tout le travail consiste alors à faire coïncider ces "moments» et à les nouer sous une seule identité.

"Nous nous souvenions, nous allions au-devant, d'un paon et nous trouvons une pivoine. Et cet étonnement inévitable n'est pas le seul: car à côté de celui-là il y en a un autre, né de la différence, non plus entre les stylisations du souvenir et la réalité, mais entre l'être que nous avons vu la dernière fois et celui qui nous apparaît aujourd'hui sous un autre angle, nous montrant un nouvel aspect. Le visage humain est vraiment comme celui du Dieu d'une théogonie orientale, toute une grappe de visages juxtaposés dans des plans différents et qu'on ne voit pas à la fois. [...] De sorte que chaque nouvelle entrevue est une espèce de redressement qui nous ramène à ce que nous avions bien vu. [...] Chaque être est détruit quand nous cessons de le voir; puis son apparition suivante est une création nouvelle, différente de celle qui l'a immédiatement précédée, sinon de toutes. [...] Dans la confrontation de notre souvenir à la réalité
2 Marcel Proust, A l'ombre des jeunes filles en fleur, Gallimard (Folio), 1972 [1919], p. 439. 
3 Id., p. 587.

4 "Je suis persuadé que c'est Albertine que je retrouve, la même que celle qui s'arrêtait souvent, au milieu de ses amies, dans sa promenade, dépassant l'horizon de la mer; mais toutes ces images restent séparées de cette autre parce que je ne peux pas lui conférer rétrospectivement une identité qu'elle n'avait pas pour moi au moment où elle a frappé mes yeux; quoi que puisse m'assurer le calcul des probabilités, cette jeune fille aux grosses joues qui me regarda si hardiment au coin de la petite rue et de la plage et par qui je crois que j'aurais pu être aimé, au sens strict du mot revoir, je ne l'ai jamais revue." id., p. 504. nouvelle, c'est cela qui marquera notre déception ou notre surprise, nous apparaîtra comme la retouche de la réalité en nous avertissant que nous nous étions mal rappelé. A son tour l'aspect la dernière fois négligé du visage, et, à cause de cela même, le plus saisissant cette fois-ci, le plus réel, le plus rectificatif, deviendra matière à rêverie, à souvenirs. »3

En outre, d'autres difficultés peuvent aussi jouer un rôle dans la perception: nuit ou conditions sombres, manque de temps pour pouvoir reconnaître la personne, ou même trouble (émotif ou psychologique) - un trouble de la reconnaissance qui est très lié à la séduction, aux ombres, à la furtivité et au désir. Qui n'a pas expérimenté cette difficulté à retrouver ou reconstituer, mentalement, les traits d'une personne dont l'apparition a été bouleversante, à un titre ou un autre? Les traits échappent ou ne se recoupent pas, ils sont parasités par d'autres souvenirs et impressions, paroles, gestes, odeurs ou éléments du contexte, et peuvent aussi être parasités par les traits d'autres personnes qui viennent s'intercaler dans les interstices, constituant une image hybride ou substituant parfois un visage à un autre (comme dans le Travail du rêve décrit par Freud). Et si cette incertitude sur les traits peut être levée par le fait de revoir la personne en question, la coïncidence n'est pourtant jamais complète entre les deux images. Cela est particulièrement clair chez Proust ${ }^{4}$ mais nous en avons des exemples dans le film de Ruiz: dans la séquence où Marcel, évoquant un souvenir ancien, dit à Gilberte qu'il n'avait pas bien vu avec qui elle se promenait ce jour-là (avec un homme ou une femme?), Gilberte propose de le lui dire, Marcel ne veut pas et de nervosité brise une tasse - et c'est aussi la reconnaissance comme action de faire coïncider qui s'en trouve brisée. Lorsque malade, Marcel rêve ou délire dans son lit, il voit une apparition qu'il nomme "Albertine", mais qui demeure à l'écran non clarifiée. Relisant une lettre, il confond les signatures d'Albertine et de Gilberte, signatures ambivalentes qui se transforment à l'écran lorsqu'un nom est proposé à la place d'un autre. Pendant une nuit de bombardements, croisant quelqu'un en arrivant près d'un bordel masculin, Marcel demande: "Cet homme, c'était Robert de Saint-Loup, ou je me trompe?». Saint-Loup, un personnage complexe avec bien des zones d'ombre, est d'ailleurs un personnage que Marcel - pourtant son très proche ami - peine à reconnaître à plusieurs reprises : toujours dans le film, lorsqu'il le voit en permission, de retour du front, il lui dit: "Et puis je ne te reconnais pas». A Balbec, enfant, regardant vers la plage depuis la terrasse, il dit "Mais, c'est Robert de Saint-Loup ou je me trompe?"; sa grand-mère ne voit rien, et un plan nous montre Saint-Loup galopant sur la plage en poussant son cri de 
guerre - le mélange des temps inscrit alors la reconnaissance dans la durée d'une vie, et même de la mort, puisque le film enchaîne directement sur l'enterrement de Saint-Loup - dont on précise qu'il avait "le nez coupé en deux", qu'il était "tout dévisagé», ce qui entame à un autre degré la reconnaissance.

\section{La question de la reconnaissance chez Ruiz}

Cependant, si la sensibilité à cette question se prête particulièrement bien à un traitement littéraire, les moyens du cinématographe sont plus limités pour en témoigner: un acteur est figé comme tel, moins glissant qu'un simple nom, un visage y est immédiatement visible, et même si on peut, par différents effets, le brouiller, le masquer, le laisser dans l'ombre ou dans la distance, voire l'interchanger, on ne peut pas transcrire les impressions décrites par Proust. Car justement, la caméra ne fait pas "d'erreur d'optique»:

"Ainsi ce n'est qu'après avoir reconnu, non sans tâtonnements, les erreurs d'optique du début qu'on pourrait arriver à la connaissance exacte d'un être si cette connaissance était possible. Mais elle ne l'est pas; car tandis que se rectifie la vision que nous avons de lui, luimême, qui n'est pas un objet inerte, change pour son compte, nous pensons le rattraper, il se déplace, et, croyant le voir enfin plus clairement, ce n'est que les images anciennes que nous en avions prises que nous avons réussi à éclaircir, mais qui ne le représentent plus.»5

Ruiz pourtant s'y confronte à plusieurs reprises dans son film. Ce n'est pas pour rien qu'il ouvre pour ainsi dire le film sur le moment des photographies que nous avons décrit en introduction. Même s'il entre moins dans cette question que Proust, et même si ce n'est sans doute pas le point central de son film, Ruiz a parfaitement conscience de son importance. Il s'y attache de différentes manières : en mettant en jeu la reconnaissance à l'intérieur du récit (tel personnage reconnaît/ ne reconnaît pas tel autre), en brouillant l'identité d'un personnage par l'image (plans enchaînés d'un visage vieux et d'un visage jeune, ou inversement, plans de dos, ou plans sombres), mais aussi en attaquant de biais par la question de la représentation (séquence des photos du début, mais aussi au travers des statues poudrées, qui reviennent à plusieurs reprises dans le film - figeant les protagonistes dans le mortuaire sous l'œil du jeune Marcel au début, et du jeune accompagné du vieux à la fin). Cette mise en crise de l'identité, qui est tension entre permanence de l'identité et évanescence de l'instant, est d'ailleurs centrale dans le film, que cela soit par la décomposition des personnages en bribes - il n'y a pas de "premiers rôles» dans le film, et chaque protagoniste est réduit à des éclats - 
6 Un effet de liquéfaction de l'image qui peut faire écho au bain photo autant qu'à une tradition "impressionniste" des jeux d'eau musicaux (Ravel, Liszt, p. ex.) ou des marines en peinture. par l'atemporalité - les contemporanéités sont glissantes tout en restant marquées - ou par la pétrification - même sans référer directement aux statues présentes dans le film, les personnages sont en quelque sorte figés dans leurs costumes et leurs décors comme des tableaux vivants.

Bien sûr, le moment central de cette question est, dans le livre comme dans le film, cette "matinée» où Marcel, revoyant vieillies ses anciennes connaissances, ne les reconnait pas - mais il serait faux de s'y réduire, et de réduire aussi la question au passage du Temps.

Dès lors, regardons donc de plus près comment cela fonctionne dans le film. Ruiz opère notamment par des plans enchaînés sur le même personnage en des temps différents: ainsi, au début du film, un plan sur Marcel et Gilberte enfants dans un jardin se mue en plan sur Gilberte adulte dans le même jardin, alors qu'elle est nommée par Marcel. Ils vont ensuite prendre la pose pour une photo de groupe: un bref plan intercalé nous remontre Marcel jeune, et un jeu optique sur l'image donne une impression d'évanescence liquide ${ }^{6}$, suivi par un nouveau plan sur Marcel jeune, et finalement par un plan sur la photo supposément obtenue (fig. 1-6). Dans cette courte séquence, nous avons trois choses: la reconnaissance, le temps et la question de la photographie, tout cela sous l'angle de l'évanescence. C'est au moment de fixer les visages en portraits (par la photo) qu'ils se troublent, et le temps avec eux. Quant à la reconnaissance (par le nom prononcé), il réunit et opère la transition entre deux temps de Gilberte, mais ces temps de Gilberte ne coïncident pas forcément avec les temps de Marcel - et si les identités sont claires, c'est alors la reconnaissance du moment qui ne l'est pas (enfance? présent de la pose? moment où l'on regarde la photo prise?).

A un autre moment, Ruiz met en scène la question de la reconnaissance à l'intérieur du récit avec un jeu sur la polysémie du mot «confondre»: au début du film, se promenant avec Marcel, Gilberte lui désigne un homme qu'ils viennent de croiser, en indiquant que c'est cet homme que son mari Saint-Loup est censé avoir rejoint à Paris. Elle ajoute: "mais je n'ai pas la force de le confondre." Plus tard, descendant l'escalier de la maison pour rejoindre Saint-Loup, elle s'est affublée des habits et du maquillage de Rachel, la maîtresse de Saint-Loup. Confondre, ici, prend ses deux sens : on "confond» Gilberte et Rachel - le film d'ailleurs nous montre tantôt le visage de l'une (Emmanuelle Béart), tantôt le visage de l'autre (Elsa Zylberstein), tandis que Gilberte «confond»Saint-Loup. Mais dans les deux cas il s'agit d'un confondre avorté : Saint-Loup ne se laisse pas prendre à ne pas la reconnaître, et Gilberte ne cherche pas à obtenir les «aveux» d'infidélité de Saint-Loup. 

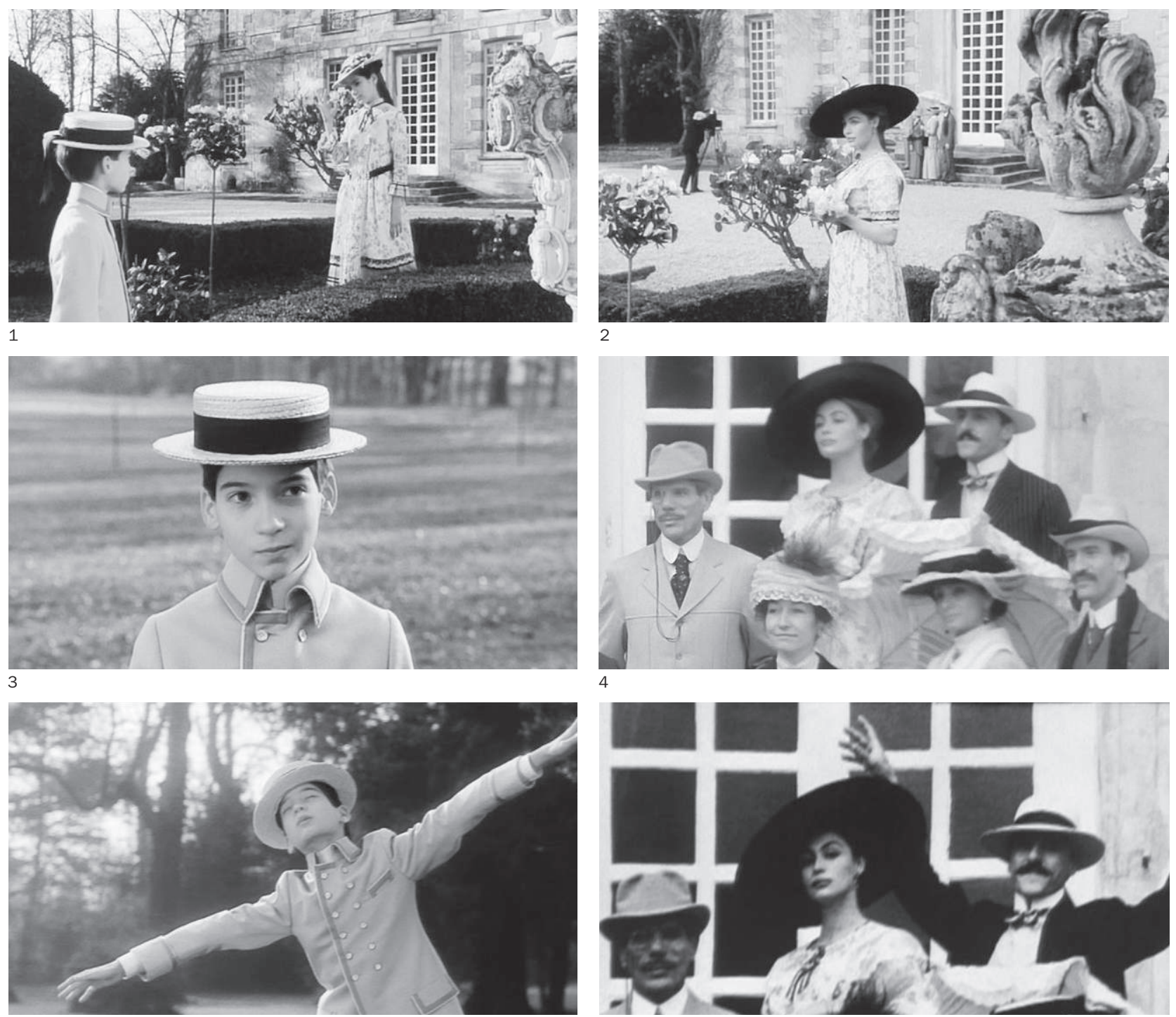

On ne compte plus par ailleurs les moments où la reconnaissance entre directement en jeu dans le film par le biais de conversations, par exemple dans la matinée finale où toutes ces reconnaissances s'enchấnent: Oriane peine à reconnaître Marcel (qui n'a pas vieilli, qui n'a pas un seul cheveu blanc), Marcel n'a pas du tout reconnu M. d'Argencourt, Cambremer demande à Marcel s'il le reconnaît (ce dernier hésite 
longtemps, puis s'exclame: "Cambremer! [...] Excusez-moi, bien sûr que je vous reconnais!»). Ensuite Gilberte, vieillie, dit à Marcel qu'il l'a sans doute prise pour sa mère (Odette) - et de fait c'est d'abord le visage d'Odette (Catherine Deneuve) que l'on voit à l'écran, vite remplacé par celui d'Emmanuelle Béart. La séquence s'achève sur $\mathrm{M}^{\mathrm{me}}$ Verdurin, vieille, qui est devenue la nouvelle Princesse de Guermantes - soulignant ainsi que même les identités et les noms sont changeants et peu fiables - avec encore un jeu de va-et-vient de plans entre elle jeune et elle vieille. A cela on peut ajouter une autre séquence dans un parc, où Charlus salue d'une révérence $\mathrm{M}^{\mathrm{me}}$ de Saint-Euverte, qu'il déteste, comme s'il ne l'avait pas reconnue.

\section{Le tour de vis}

Cette question centrale dans le livre est donc traitée par petites touches dans le film, même si Ruiz en mesure l'importance. Mais il utilise des moyens cinématographiques finalement assez grossiers, les plans enchấnés des visages différents demeurant la principale forme de l'expression du trouble de la reconnaissance. On pourrait être assez déçu, d'autant qu'en adepte d'une "esthétique de l'ombre", il aurait pu beaucoup plus jouer avec cela - il ne le fait guère qu'avec le personnage de Charlus. C'est par le tour de vis supplémentaire de la reconnaissance acteur/ personnage, activée par le spectateur, que le film devient intéressant sur ce point.

Car c'est parfois directement pour le spectateur que Ruiz met en jeu la question de la reconnaissance, en faisant appel aux souvenirs du lecteur de Proust et à ce que celui-ci pourra reconnaître de sa lecture dans le film (en particulier la coïncidence d'un acteur avec un personnage). Car pour le spectateur qui a lu Proust, le jeu de la reconnaissance prend un autre tour encore. Qui joue quel personnage? Vais-je «reconnaître» celui constitué par mon imaginaire à la lecture de Proust? Sans compter qu'on ne "reconnaît" pas les acteurs eux-mêmes, tant Ruiz est un excellent directeur d'acteur: même Emmanuelle Béart et Vincent Perez, au jeu d'ordinaire assez limité, sont ici excellents. Ruiz s'amuse et prend plaisir à faire travailler la reconnaissance par le spectateur. Ainsi la présentation à l'écran de Charlus (personnage attendu s'il en est par tout lecteur de Proust) est différée et repoussée par divers effets à la fois de construction narrative et de mise en scène. Quant au personnage d'Oriane, son exposition à l'enterrement de Saint-Loup est exemplaire : alors que les personnages en marche défilent devant la caméra, quittant le cimetière, la caméra s'attarde alors sur un personnage que l'on ne connaît pas encore, qui s'arrête juste dans le champ; un homme vient 
alors l'appeler et fait sortir cette personne du champ: «Venez, Oriane, venez". Là encore, il s'agit d'un personnage majeur et attendu de tout lecteur de la Recherche, même s'il n'a qu'un rôle mineur dans le film.

D'une certaine manière, Proust ne décrit pas des personnages, il décrit les impressions provoquées par ces personnages. Cette finesse de subjectivité est plus puissante que toute description naturaliste ou réaliste - combien de fois un personnage, décrit comme petit ou blond par un roman, se retrouve grand ou noiraud dans l'imagination du lecteur? En déplaçant la précision de l'objectif vers le subjectif, Proust laisse une grande liberté imaginative au lecteur concernant les traits, et se concentre sur l'essentiel, à savoir ce qui émane des personnages. Ruiz en est conscient: si l'on reconnaît les personnages de la Recherche dans son film, ce n'est pas tant qu'ils correspondent à des descriptions physiques - rien à voir avec le travail préparatoire d'un Peter Jackson pour Le Seigneur des Anneaux (The Lord of the Rings, Etats-Unis, 2001-2003), autre livre réputé inadaptable $\mathbf{7}$-, mais parce qu'ils sont constitués sur la base d'impressions, et aussi qu'ils émanent - dans le film comme dans le livre - d'une voix à la fois extérieure et intérieure au récit, celle de Marcel. Le spectateur n'a alors plus à reconnaître des traits physiques, mais des impressions ressenties et imaginées, qu'il confronte alors avec celles dégagées par le film, avec succès ou non, les rectifiant ou non, rejouant alors les impressions décrites par Proust à un degré supplémentaire... En passant du côte de la fiction, l'acte de reconnaissance déjoue également l'idée de mort qui lui est attachée, et prête alors aux personnages une éternité rejouée - ou même enjouée.
7 Avant de lancer son film, Peter Jackson avait ouvert une vaste consultation sur internet pour savoir comment les lecteurs de Tolkien imaginaient l'aspect des différents personnages et comment ils souhaitaient les voir à l'écran. 